\title{
Delivered Dose Verification for Lung Cancer Stereotactic Body Radiotherapy Using Cone-Beam CT
}

\author{
Yechao Feng ${ }^{*}$, Xi Liu ${ }^{2,3 *}$, Xiang Pan ${ }^{4 *}$, Chang Hu${ }^{5}$, Ning Zhan6, Zongda Zhu6 ${ }^{6}$, Zhenxiang Deng6\# \\ ${ }^{1}$ Department of Radiation Oncology, Hunan Academy of Traditional Chinese Medicine Affiliated Hospital, Changsha, China \\ ${ }^{2}$ School of Medical Information and Engineering, Guangdong Pharmaceutical University, Guangzhou, China \\ ${ }^{3}$ Guangdong Province Precise Medicine and Big Data Engineering Technology Research Center for Traditional Chinese Medicine, \\ Guangzhou, China \\ ${ }^{4}$ Department of Radiation Oncology, Yunnan Tumor Hospital, The Third Affiliated Hospital of Kunming Medical University, \\ Kunming, China \\ ${ }^{5}$ The Second Affiliated Hospital of Hainan Medical University, Haikou, China \\ ${ }^{6}$ Department of Radiation Oncology, School of Medicine, Xiang'an Hospital of Xiamen University, Xiamen University, Xiamen, \\ China \\ Email: \#ghost_fx135@163.com
}

How to cite this paper: Feng, Y.C., Liu, X., Pan, X., Hu, C., Zhan, N., Zhu, Z.D. and Deng, Z.X. (2021) Delivered Dose Verification for Lung Cancer Stereotactic Body Radiotherapy Using Cone-Beam CT. Journal of Cancer Therapy, 12, 453-459. https://doi.org/10.4236/jct.2021.127039

Received: June 28, 2021

Accepted: July 23, 2021

Published: July 26, 2021

Copyright $\odot 2021$ by author(s) and Scientific Research Publishing Inc. This work is licensed under the Creative Commons Attribution International License (CC BY 4.0).

http://creativecommons.org/licenses/by/4.0/ (c) (i) Open Access

\begin{abstract}
Purpose: Verified the delivered dose distribution of lung cancer Stereotactic Body Radiotherapy (SBRT) using the cone-beam CT images. Methods: Twenty lung cancer patients who underwent SBRT with $100 \mathrm{CBCT}$ images were enrolled in this study. Delivered dose distributions were recalculated on CBCT images with the deformed and non-deformed methods, respectively. The planned and delivered dose distributions were compared using the dose-volume histograms. Results: The delivered target coverage (V100) per patient inside target volume deviated on average were $0.83 \% \pm 0.86 \%$ and $1.38 \% \pm 1.40 \%$ for Pct $v s$. Pcbct and Pct vs. Pdcbct, respectively. The Conformity Index (CI) and Gradient Index (GI) showed a good agreement among the plans. For the critical organs, only minor differences were observed between the planned dose and the delivered dose. Conclusions: CBCT images were a useful tool for setup and dose delivery verification for lung cancer patients who underwent SBRT.
\end{abstract}

\section{Keywords}

Lung Cancer, Stereotactic Body Radiotherapy, Cone Beam CT, Dose Verification 


\section{Introduction}

Currently, Stereotactic Body Radiation Therapy (SBRT) has been widely used in the treatment of stage I/II medical inoperable and surgically unresectable nonsmall-cell and metastatic lung cancer in recent years [1]-[6]. The treatment allows delivery of a very high dose of radiation in a few fractions to small regions (hypo-fractionated). Therefore, an accurate dose delivery is crucial for a successful SBRT. Due to the effect of breathing motion on small targets volumes is more severe, steep dose gradients and inhomogeneous densities in the thoracic region, accurate treatment delivery is not always guaranteed [7] [8]. Hence, dose delivery verification is a prerequisite to assure correct treatment planning and delivery for SBRT treatment.

Over the past years, the kilovoltage Cone-Beam-Compute Tomography (CBCT) system attached to a linear accelerator has become commercially available for Image-Guide Radiotherapy (IGRT) [9]. The CBCT images are useful for verifying not only the position of a treated tumor but also the regression or progression of the tumor. Optimal radiation therapy may be accomplished with regard to the field margin related to change in the target size and position, as well as the location of critical organs on every treatment day [10] [11]. That is, a dose distribution that takes account of any modification of the targeted region is calculated at each radiation therapy session [12] [13].

At the time of treatment, the patient's anatomy is imaged with the current in-room capabilities. We have used the $\mathrm{kV} \mathrm{CBCT}$ imaging currently available on our treatment machine. Recently, the Philips Pinnacle treatment planning system provides a dynamic planning module. It has two implementation methods: one is only copying all the structures from the original image to the second image; the other is copying the structures to the second image according to the deformable registration. The delivered dose in the patient anatomy is then re-optimization in CBCT images using the Pinnacle dynamic planning module. This procedure provides 3D dose verification in the patient anatomy during a treatment fraction.

The aim of this study was to verify the delivered dose distribution of lung cancer Stereotactic Body Radiotherapy (SBRT) using the cone-beam CT images.

\section{Materials and Methods}

For this study, we retrospectively selected twenty non-small cell lung cancer patients treated in our clinic with SBRT, which every fraction the CBCT image can be used dose calculation. Patients were immobilized using Body Fix system (Elekta, Crawley, UK) to improve positioning reproducibility and to reduce the target motion with arms placed on their forehead. For these patients, a 4D CT image was scanned with a Philips Brilliant spiral CT (Philips Brilliant, Cleveland, $\mathrm{OH}$ ) according to standard procedures with $1 \mathrm{~mm}$ slice spacing. The CBCT image was acquired before every fraction treatment using the Elekta Synergy which equipped the kv cone-beam CT. 


\subsection{Treatment Planning}

All 4D CT and CBCT datasets were transferred into a commercial treatment planning system (Pinnacle 9.8, Philips). The Gross Target Volume (GTV) was contoured by a senior radiotherapy oncologist using standardized lung window level setting. The amplitude of tumor motion was acquired from the 4D CT scan and incorporated as a patient-specific margin in the Internal Target Volume (ITV). The ITV to Planning Target Volume (PTV) margin was fixed in $3 \mathrm{~mm}$.

Two hundred and twenty full arc or partial arc Volumetric Modulated Arc Therapy (VMAT) plans were generated using the Pinnacle 9.8 Treatment Planning System (TPS) (Philips). It consisted of twenty delivered plans on CT (Pct), 100 recalculated plans with deformable mapping (Pdcbct) and 100 plans non-deformable mapping on CBCT (Pcbct), respectively. For the Pct plans, the double full arc or partial arc plans were generated according the tumor position. For the sake of dosimetirc comparison, prescription was normalized to $50 \mathrm{~Gy}$ at 5 fractions at $6 \mathrm{MV}$ for the all plans. The plans were optimized to reach clinically acceptable PTV coverage and Organ at Risk (OAR) sparing and normalized so that $95 \%$ of the PTV was covered by $100 \%$ of the prescription dose.

\subsection{Dosimetric Comparison}

Several metrics were used for plan comparison. The target coverage (V100\%) was defined the PTV volume receiving of Prescribed Dose (PD). The Conformity Index (CI) was defined as the ratio of the volume of the $100 \%$ isodose line (V100) to the target volume (PTV): CI = V100/PTV. The Gradient Index (GI) was defined as the ratio of the $50 \%$ isodose line (V50) volume to the target volume (PTV): GI = V50/PTV. For the health tissue, the V10 Gy, V15 Gy, V20 Gy of lungs, D2 of the cord, V20 Gy and V30 Gy of chest wall were evaluated compared among the Pct, Pdcbct and Pcbct plan.

\subsection{Statistic}

Results were described as mean \pm Standard Deviation (SD). Comparisons among the plans were analyzed with one-way ANOVA method. All statistical analysis was conducted with $\mathrm{R}$ program software. Difference was considered statistically significant when $\mathrm{p}<0.05$.

\section{Results}

Table 1 shows the partial dosimetric comparison among the Pct, Pcbct, Pdcbct. The target coverage (V100) per patient inside target volume deviated on average were $0.83 \% \pm 0.86 \%$ and $1.38 \% \pm 1.40 \%$ for Pct $v s$. Pcbct and Pct vs. Pdcbct, respectively. One-way ANOVA analysis found only one patient have a significance difference among the three Pct, Pcbct, Pdcbct ( $\mathrm{p}$ value $<0.05$ ). The Conformity Index (CI) and Gradient Index (GI) showed a good agreement between the planned and delivered dose distribution for patients without changes in anatomy. The one-way ANOVA results showed there have no significance difference; the $\mathrm{p}$ value was $0.73 \pm 0.09$ and $0.58 \pm 0.22$ for $\mathrm{CI}$ and GI, respectively. 
Table 1. Target parameter comparison.

\begin{tabular}{|c|c|c|c|c|c|c|c|}
\hline Objectives & & Pct & Pcbct & Pdcbct & Pct vs. Pcbct (\%) & Pct vs. Pdcbct (\%) & $P$ value \\
\hline \multirow{5}{*}{ PTV_100 (5 patients) } & Patient 1 & 95.46 & $94.5 \pm 1.84$ & $95.14 \pm 0.81$ & 2.03 & 4.11 & $<0.05$ \\
\hline & Patient 2 & 94.45 & $94.5 \pm 1.84$ & $95.14 \pm 0.81$ & 0.05 & 0.73 & 0.6 \\
\hline & Patient 3 & 95.35 & $95.46 \pm 0.58$ & $95.1 \pm 1.24$ & 0.11 & 0.26 & 0.77 \\
\hline & Patient 4 & 95.51 & $95.30 \pm 1.12$ & $94.52 \pm 2.57$ & 0.22 & 1.03 & 0.61 \\
\hline & Patient 5 & 96.18 & $95.50 \pm 0.20$ & $96.45 \pm 1.14$ & 0.71 & 0.29 & 0.11 \\
\hline \multirow{5}{*}{ CI (5 patients) } & Patient 1 & 1.02 & $1.02 \pm 0.01$ & $1.01 \pm 0.04$ & 0 & 0.98 & 0.76 \\
\hline & Patient 2 & 0.97 & $0.96 \pm 0.03$ & $0.96 \pm 0.02$ & 1.03 & 1.03 & 0.74 \\
\hline & Patient 3 & 1.04 & $1.03 \pm 0.02$ & $1.04 \pm 0.02$ & 0.96 & 0 & 0.8 \\
\hline & Patient 4 & 1.03 & $1.04 \pm 0.03$ & $1.03 \pm 0.03$ & 0.97 & 0 & 0.78 \\
\hline & Patient 5 & 1.08 & $1.08 \pm 0.02$ & $1.07 \pm 0.02$ & 0 & 0.93 & 0.55 \\
\hline \multirow{5}{*}{ GI (5 patients) } & Patient 1 & 4.16 & $4.16 \pm 0.06$ & $4.13 \pm 0.1$ & 0 & 0.72 & 0.66 \\
\hline & Patient 2 & 5.37 & $5.36 \pm 0.3$ & $5.44 \pm 0.21$ & 0.19 & 1.3 & 0.82 \\
\hline & Patient 3 & 3.86 & $3.83 \pm 0.08$ & $3.78 \pm 0.09$ & 0.78 & 2.07 & 0.18 \\
\hline & Patient 4 & 3.78 & $3.70 \pm 0.2$ & $3.77 \pm 0.21$ & 2.12 & 0.26 & 0.7 \\
\hline & Patient 5 & 5 & $4.96 \pm 0.13$ & $5.03 \pm 0.1$ & 0.8 & 0.6 & 0.55 \\
\hline
\end{tabular}

Table 2 lists the partial OARs protection comparison among the three planning modalities. In this table, we observed all the metrics have a significant difference in the patient 1 . In others patients, only a few of the metrics have a significant difference.

\section{Discussion}

Treatment verification using CBCT images based on information acquired in the treatment room is feasible and provides an independent verification for lung cancer SBRT patients. In this study, we retrospectively investigated the delivery dose effect of without deformable and deformable planning on twenty lung SBRT patients. We have analyzed the twenty patient cases and two hundred and twenty plans included 20 Pct, 100 Pdcbct, 100 Pcbct plans. We found the volume of PTV in the three modalities is essentially the same. This was expected, because for these patients only small changes in anatomy were observed compared to the planning CT image. For the PTV metrics, we just found the V100 has a significant difference in one patient, the CI and GI didn't find any significant difference for the patients. It shows that the treatment plan in most patients can get an exact delivery.

There have several advantages for dose verification using the information acquired prior to a treatment session. First, on the first day of treatment, the patient anatomy is scanned with an in-room image-guided radiotherapy technique: in this study $\mathrm{kV}$ cone-beam CT imaging. The treatment planning CT (4D) image was acquired several days or weeks prior to the first fraction. The patient's anatomy 
Table 2. OAR dosimetric comparison.

\begin{tabular}{|c|c|c|c|c|c|c|c|c|}
\hline \multirow{2}{*}{\multicolumn{2}{|c|}{ Objectives }} & \multicolumn{3}{|c|}{ Lung } & \multirow{2}{*}{$\begin{array}{c}\text { Cord } \\
\text { D2 (Gy) }\end{array}$} & \multicolumn{3}{|c|}{ Chest wall } \\
\hline & & V10 Gy (cc) & V15 Gy (cc) & V20 Gy (cc) & & V20 Gy (cc) & V30 Gy (cc) & V40 Gy (cc) \\
\hline \multirow{4}{*}{ Patient 1} & Pct & 411.52 & 297.9 & 222.27 & 1294.38 & 54.71 & 25.19 & 4.65 \\
\hline & Pcbct & $503.42 \pm 10.42$ & $365.31 \pm 20.89$ & $289.45 \pm 16.84$ & $1354.64 \pm 84.8$ & $50.96 \pm 0.51$ & $23.34 \pm 0.08$ & $5.67 \pm 0.84$ \\
\hline & Pdcbct & $553.02 \pm 23.46$ & $384.64 \pm 8.96$ & $310.53 \pm 9.16$ & $1444.7 \pm 27.38$ & $33.7 \pm 1.81$ & $19 \pm 1.07$ & $5.83 \pm 0.53$ \\
\hline & $\mathrm{P}$-value & $<0.05$ & $<0.05$ & $<0.05$ & $<0.05$ & $<0.05$ & $<0.05$ & 0.05 \\
\hline \multirow{4}{*}{ Patient 2} & Pct & 361.36 & 239.88 & 165.12 & 969.78 & 49.09 & 14.55 & 3.55 \\
\hline & Pcbct & $356.12 \pm 13.08$ & $245.09 \pm 8.12$ & $180.30 \pm 15.12$ & $917.31 \pm 48.88$ & $49.42 \pm 0.77$ & $14.59 \pm 0.08$ & $3.68 \pm 1.15$ \\
\hline & Pdcbct & $378.80 \pm 63.51$ & $240.49 \pm 30.08$ & $195.46 \pm 19.32$ & $949.78 \pm 166.25$ & $49.21 \pm 0.52$ & $14.48 \pm 0.14$ & $3.54 \pm 0.85$ \\
\hline & $\mathrm{P}$-value & 0.58 & 0.46 & 0.24 & 0.71 & 0.63 & 0.22 & 0.96 \\
\hline \multirow{4}{*}{ Patient 3} & Pct & 263.24 & 149.72 & 105.24 & 1095.24 & 19.36 & 16.39 & 9.05 \\
\hline & Pcbct & $270.64 \pm 56.13$ & $140.23 \pm 16.04$ & $120 \pm 7.19$ & $1081.9 \pm 97.2$ & $19.38 \pm 0.56$ & $16.36 \pm 0.06$ & $8.72 \pm 0.5$ \\
\hline & Pdcbct & $255.35 \pm 40.78$ & $164.35 \pm 26.15$ & $118.15 \pm 4.15$ & $1091.48 \pm 177.17$ & $19.2 \pm 0.62$ & $16.44 \pm 0.1$ & $8.43 \pm 1.34$ \\
\hline & $\mathrm{P}$-value & $<0.05$ & 0.14 & 0.12 & 0.98 & 0.82 & 0.22 & 0.51 \\
\hline \multirow{4}{*}{ Patient 4} & Pct & 378.63 & 259.28 & 177.64 & 579.24 & 53.74 & 13.78 & 1.33 \\
\hline & Pcbct & $400.34 \pm 20.18$ & $264.15 \pm 31.12$ & $170.57 \pm 19.34$ & $549.37 \pm 142.03$ & $53.49 \pm 0.68$ & $13.73 \pm 0.07$ & $1.22 \pm 0.56$ \\
\hline & Pdcbct & $394.43 \pm 51.21$ & $278.12 \pm 21.73$ & $195.07 \pm 17.76$ & $607.17 \pm 116.6$ & $54.03 \pm 0.4$ & $13.81 \pm 0.08$ & $0.62 \pm 0.73$ \\
\hline & $\mathrm{P}$-value & 0.8 & $<0.05$ & 0.14 & 0.7 & 0.21 & 0.13 & 0.11 \\
\hline \multirow{4}{*}{ Patient 5} & Pct & 451.48 & 281.84 & 215.12 & 1025.86 & 85.21 & 48.22 & 17.6 \\
\hline & Pcbct & $460.14 \pm 35.16$ & $289.42 \pm 24.87$ & $210.14 \pm 9.14$ & $1036.21 \pm 118.58$ & $85.41 \pm 0.92$ & $48.25 \pm 0.09$ & $16.92 \pm 0.74$ \\
\hline & Pdcbct & $473.64 \pm 16.47$ & $280.78 \pm 18.45$ & $220.87 \pm 15.45$ & $1054.05 \pm 67.11$ & $85.48 \pm 0.53$ & $48.21 \pm 0.17$ & $17.24 \pm 1$ \\
\hline & P-value & $<0.05$ & 0.88 & 0.52 & 0.85 & 0.78 & 0.78 & 0.36 \\
\hline
\end{tabular}

may occur some changes in this period. If these possible changes really happen, it is visible in the CBCT image acquired for the IGRT procedure used for patient set-up. In Table 1 , we can find the changes are very small. In our institution, only a $3 \mathrm{D}$ imaging technique (3D CBCT) is available. The results may be improved with a $4 \mathrm{D}$ CBTC technique, not only for the anatomy changes but also for the possible changes in the breathing motion [14]. Second, we generated the plans used the same beam parameter, optimization parameter on the fraction CBCT with the dynamic module of the pinnacle TPS. It has two ways to implementation, one is to put the structures from the original CT images to the CBCT images without any deformable, the other is to put the structures from the original CT images to the $\mathrm{CBCT}$ images depend on the deformable registration, it also called adaptive radiotherapy. In these three modalities plan, the contours only delineated in the original CT and the beam and optimization parameters were the same, therefore the difference of the manufactured could be decreased as far as possible.

The feasibility of CBCT to calculation dose has been previously investigated. 
Yoo et al. [13] studied the Hounsfield Unit (HU) value difference between CT and CBCT images for the Catphan phantoms (The Phantom Laboratory, NY). Yang et al. [12] evaluated the dose difference between the CT plan and CBCT plan; it found the dose difference between the two was within $2 \%$. However, due to the respiratory motion, the dose differences can be higher (3\%) in the lungs. Both studies suggest that $\mathrm{CBCT}$ can be employed directly in dose calculation.

In this study, the target coverage $(\mathrm{V} 100 \%)(\mathrm{p}<0.01)$ and HI $(\mathrm{p}=0.01)$ had a significant difference in patient 1 , and not observed any significant differences in other patients. From Table 1, we found the volume of PTV was higher than the other four patients. Qin et al. [15] studied the target size change has been shown an important metric determining the dosimetric effects of adaptive planning for lung SBRT, and the patients with small target size changes are less likely to profit from adaptive planning due to anticipated small dosimetric changes. The CI and GI were not found any significant differences for all the patients.

The 3D dose verification is to add up the dose distributions of the various fractions. For this purpose, non-deformable and deformable mappings are needed. From the results, we found the planning dose could be got a better delivery. For the OARs, the large volume of the PTV the delivered dose has a significant difference, while the smaller volume of PTV has no significant difference. Therefore, an adaptive lung SBRT should be better for the larger target volumes to reduce to delivery dose.

\section{Funding}

The study was partially supported by a grant from Guangdong Province innovation school project NO. 2018KQNCX128, Xiamen Medical and Health Guidance Project NO. $3502 Z 20209111$.

\section{Conflicts of Interest}

The authors declare no conflicts of interest regarding the publication of this paper.

\section{References}

[1] Fakiris, A.J., McGarry, R.C., Yiannoutsos, C.T., Papiez, L., Williams, M., Henderson, M.A. and Timmerman, R. (2009) Stereotactic Body Radiation Therapy for Early-Stage Non-Small-Cell Lung Carcinoma: Four-Year Results of a Prospective Phase II Study. International Journal of Radiation Oncology·Biology. Physics, 75, 677-682. https://doi.org/10.1016/j.ijrobp.2008.11.042

[2] Guckenberger, M., Wilbert, J., Krieger, T., Richter, A., Baier, K., Meyer, J. and Flentje, M. (2007) Four-Dimensional Treatment Planning for Stereotactic Body Radiotherapy. International Journal of Radiation Oncology.Biology. Physics, 69, 276-285. https://doi.org/10.1016/j.ijrobp.2007.04.074

[3] Joyner, M., Salter, B.J., Papanikolaou, N. and Fuss, M. (2006) Stereotactic Body Radiation Therapy for Centrally Located Lung Lesions. Acta Oncologica, 45, 802-807. https://doi.org/10.1080/02841860600915322

[4] Nagata, Y., Negoro, Y., Aoki, T., Mizowaki, T., Takayama, K., Kokubo, M., et al. (2002) 
Clinical Outcomes of 3D Conformal Hypofractionated Single High-Dose Radiotherapy for One or Two Lung Tumors Using a Stereotactic Body Frame. International Journal of Radiation Oncology.Biology. Physics, 52, 1041-1046. https://doi.org/10.1016/S0360-3016(01)02731-6

[5] Okunieff, P., Petersen, A.L., Philip, A., Milano, M.T., Katz, A.W., Boros, L., Schell, M.C. (2006) Stereotactic Body Radiation Therapy (SBRT) for Lung Metastases. Acta Oncologica, 45, 808-817. https://doi.org/10.1080/02841860600908954

[6] Onishi, H., Araki, T., Shirato, H., Nagata, Y., Hiraoka, M., Gomi, K., et al. (2004) Stereotactic Hypofractionated High-Dose Irradiation for Stage I Nonsmall Cell Lung Carcinoma: Clinical Outcomes in 245 Subjects in a Japanese Multiinstitutional Study. Cancer, 101, 1623-1631. https://doi.org/10.1002/cncr.20539

[7] Knöös, T., Wieslander, E., Cozzi, L., Brink, C., Fogliata, A., Albers, D., Nyström, H. and Lassen, S. (2006) Comparison of Dose Calculation Algorithms for Treatment Planning in External Photon Beam Therapy for Clinical Situations. Physics in Medicine \& Biology, 51, 5785-5807. https://doi.org/10.1088/0031-9155/51/22/005

[8] Papanikolaou, N., Battista, J., Boyer, A., Klein, E. and Sharpe, M. (2004) Tissue Inhomogeneity Corrections for Megavoltage Photon Beams. AAPM Report 85, Task Group No. 65 of the Radiation Therapy Committee of the American Association of Physicists in Medicine, Medical Physics Publishing, Madison.

[9] Bissonnette, J.P., Purdie, T.G., Higgins, J.A., Li, W. and Bezjak, A. (2009) ConeBeam Computed Tomographic Image Guidance for Lung Cancer Radiation Therapy. International Journal of Radiation Oncology.Biology.Physics, 73, 927-934. https://doi.org/10.1016/j.ijrobp.2008.08.059

[10] Ding, G.X., Duggan, D.M., Coffey, C.W., Deeley, M., Hallahan, D.E., Cmelak, A. and Malcolm, A. (2007) A Study on Adaptive IMRT Treatment Planning Using kV Cone-Beam CT. Radiotherapy Oncology, 85, 116-125.

https://doi.org/10.1016/j.radonc.2007.06.015

[11] Jeasen, H.R., Hansen, O., Hjelm-Hansen, M. and Brink, C. (2007) Inter- and Intrafractional Movement of the Tumor in Extra Cranial Stereotactic Radiotherapy of NSCLC. Acta Oncologica, 47, 1432-1437.

https://doi.org/10.1080/02841860802251567

[12] Yang, Y., Schreibmann, E., Li, T., Wang, C. and Xing, L. (2007) Evaluation of On-Board $\mathrm{kV}$ Cone Beam CT (CBCT)-Based Dose Calculation. Physics in Medicine \& Biology, 52, 685-705. https://doi.org/10.1088/0031-9155/52/3/011

[13] Yoo, S. and Yin, F.-F. (2006) Dosimetric Feasibility of Cone-Beam CT-Based Treatment Planning Compared to Ct-Based Treatment Planning. International Journal of Radiation Oncology·Biology Physics, 66, 1553-1561.

https://doi.org/10.1016/j.ijrobp.2006.08.031

[14] Sonke, J.J., Lebesque, J. and van Herk, M. (2008) Variability of Four-Dimensional Computed Tomography Patient Models. International Journal of Radiation Oncology·Biology.Physics, 70, 590-598. https://doi.org/10.1016/j.ijrobp.2007.08.067

[15] Qin, Y., Zhang, F., Yoo, D.S., Kelsey, C.R., Yin, F.F. and Cai, J. (2013) Adaptive Stereotactic Body Radiation Therapy Planning for Lung Cancer. International Journal of Radiation Oncology.Biology. Physics, 87, 209-215.

https://doi.org/10.1016/j.ijrobp.2013.05.008 